\title{
Denoising of Time-Density Data in Digital Subtraction Angiography
}

\author{
Hrvoje Bogunović and Sven Lončarić \\ Faculty of Electrical Engineering and Computing, \\ University of Zagreb, Unska 3, 10000 Zagreb, Croatia \\ $\{$ hrvoje.bogunovic, sven.loncaric\}@fer.hr \\ http://ipg.zesoi.fer.hr
}

\begin{abstract}
In this paper we present methods for removing the noise from a time sequence of digitally subtracted x-ray angiographic images. By observing the contrast agent propagation profile in a region of the angiogram one can estimate the time of arrival of that agent. Due to the large level of noise, it is difficult to detect the moment of the contrast agent arrival accurately. Hence denoising is required. Two methods are presented. The first one is based on 1D Wiener filtering of the time data. Wiener filter was chosen because it presents the optimal linear filter in the least-squares sense. The other method is based on 3D wavelet denoising via wavelet shrinkage technique, which is a nonlinear method. Since it is based on 3D wavelet basis it can perform denoising simultaneously in the spatial as well as in the time dimension of the image sequence. Wavelet based denoising proved to be superior but computationally more demanding. The experiments were performed on a sequence of cerebral angiograms.
\end{abstract}

\section{Introduction}

Digital subtraction angiography (DSA) is often used to assess the structure of vessels. By injecting the contrast agent, which attenuates the x-rays, the vessel structure in the observed region of interest (ROI) becomes visible. Instead of viewing just the morphology, DSA can also be used to detect e.g. time of arrival of the injected agent to different regions of the tissue. This information can prove vital in detecting and locating the infarcted regions.

For acquiring the necessary data for later processing, the image acquisition process is defined. It consists of the following steps. First, contrast agent is injected into the main feeding artery. Since we are acquiring images of the brain, contrast agent in our case was injected into one of the carotids so only one brain hemisphere becomes visible. By using the high frame rate, we can get an image sequence showing propagation of the contrast agent. One image from a sequence where contrast agent is visible is shown in Fig. 1 In order to estimate the exact moment of agent arrival one needs to observe the bolus traversal time profile in the ROI. Such time-density signal is usually too noisy for the straightforward estimation of the arrival time, hence denoising of the time-density data is necessary. 


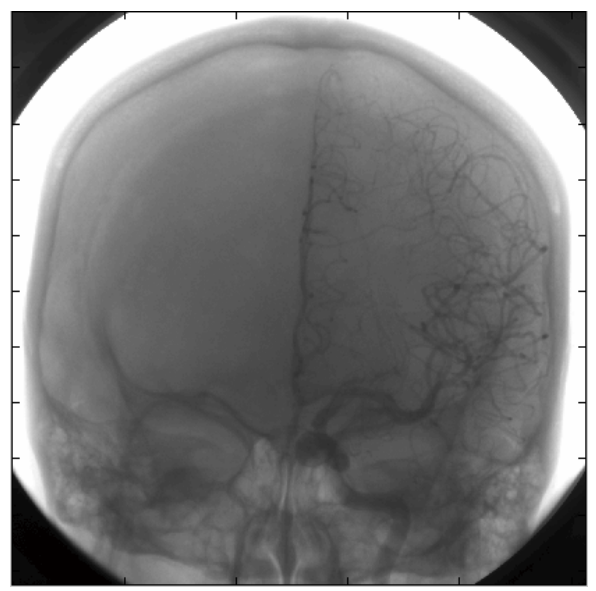

Fig. 1. AP view showing contrast agent in the left hemisphere

We made the first attempt by using the Wiener filtering approach for smoothing the 1D signal which presents the time-density curve of a ROI. Later we used a different approach by using the 3D wavelet decomposition and performed the nonlinear denoising by wavelet shrinkage.

The outline of the paper is as follows. Background about the digital subtraction angiography is given in section 2. Time-density curves and time of arrival estimation are explained in section 3. Denoising techniques are discussed in section 4 , which is the central part of the paper. Results and comparison of the used techniques are given in section 5 and section 6 concludes the paper.

\section{Digital Subtraction Angiography}

The product of the X-ray imaging device is an image showing both the enhanced vessel as well as the surrounding structure such as e.g. the bones. It is difficult to observe the vessels in such an image. Also the intensity value in the vessel is not proportional just to the amount of the contrast agent but also the structures behind the vessel contribute to the resulting intensity value due to X-ray device's projective nature.

To enhance the contrast agent visibility it is first necessary to eliminate background information from each image. This is performed using the digital subtraction from the mask image which is taken prior to the contrast injection and which shows only the non-vessel structure. This technique is called digital subtraction angiography. Before subtraction takes place both images are logarithmized to eliminate the thickness of the human body [1].

$$
\begin{aligned}
& I_{\text {mask }}=I_{0} e^{-\mu_{t} x_{t}} \\
& I_{\text {contrast }}=I_{0} e^{-\left(\mu_{t} x_{t}+\mu_{I} x_{I}\right)} \\
& S=\ln \left(I_{\text {mask }}\right)-\ln \left(I_{\text {contrast }}\right)=\mu_{I} x_{I}
\end{aligned}
$$




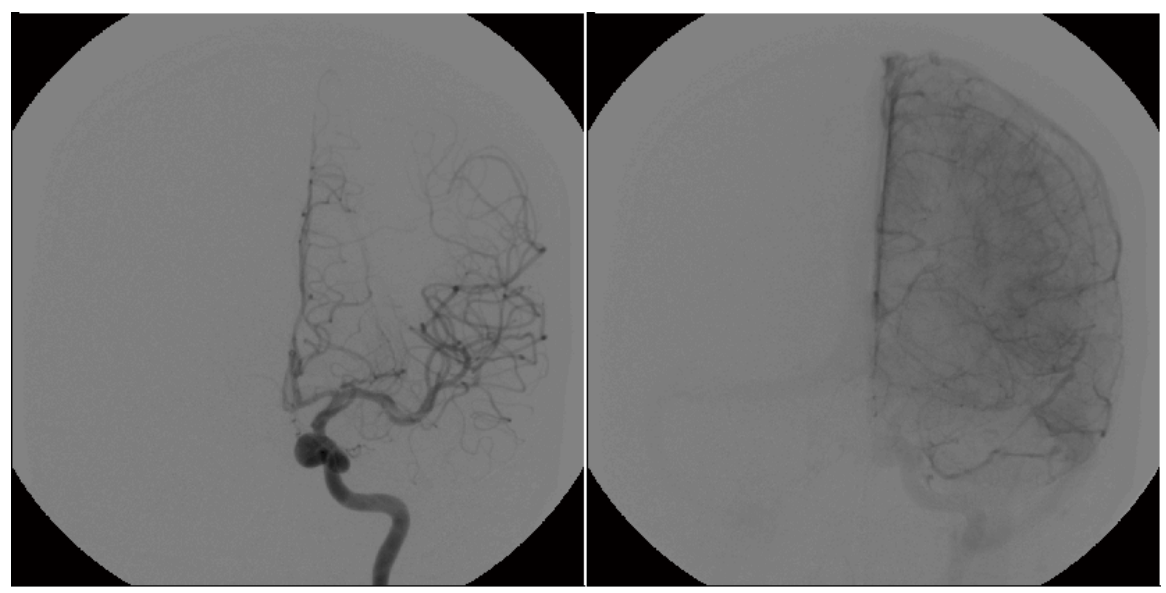

Fig. 2. Two DSA frames showing contrast propagation

Where $x_{t}$ is the thickness of the body, $x_{I}$ is the thickness of the vessel filled with contrast agent and $\mu_{t}, \mu_{I}$ are the corresponding attenuation coefficients. The intensity value of the subtracted image $S$ is proportional only to the amount of contrast in a region represented by a pixel.

In order to remove the unnecessary anatomy the mask image has to align accurately with the image containing the agent. The alignment can be affected by the motion of the patient, thus motion compensation normally precedes the subtraction. In our test images, motion of the head was negligible so no compensation was performed.

The result of DSA operation is a sequence of images showing only the contrast propagation. Two frames at different time instant are shown in Fig. 2, This sequence is used as the input image in the later parts of our procedure.

\section{Time-Density Signals}

The pixel value of the input image in this stage of the procedure is proportional to the amount of the contrast agent in that region. For constant region volume this can be interpreted like the pixel value is proportional to the density of contrast agent in the volume. When we observe one region through time we can draw a time-density curve. Idealistic view of the time-density curve for the region, which is traversed by the contrast agent is shown in Fig. 3. Propagation of the contrast agent is clearly visible in the figure. Time of arrival (TA) is depicted as time from start of the sequence till the contrast reaches its maximum density value. This was taken to be analogous to time of arrival parameter commonly used in CT measurements 2.

At the end we want to produce as a result an image, representing the whole angiogram sequence, showing the time of arrival for each region where each region is commonly represented by a pixel. 


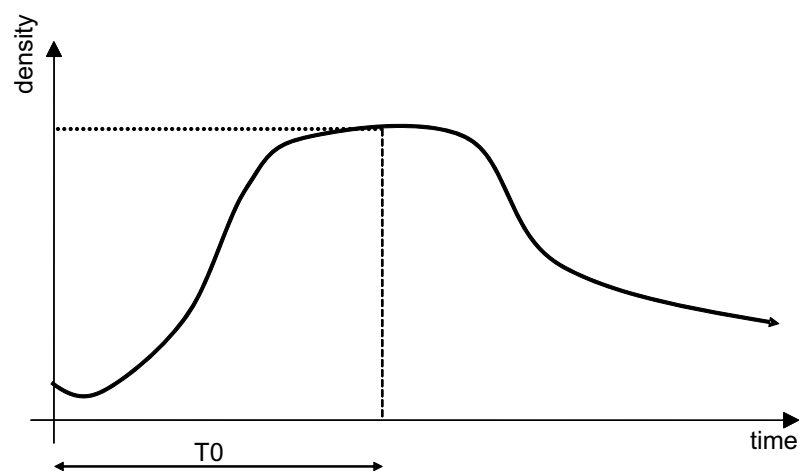

Fig. 3. Time-density signal

\section{Denoising}

In reality a time-density signal is not as smooth as it was previously shown in Fig. 3. Noise is present in the image sequence and it also appears in the curve. Additionally, due to the projective nature of X-ray imaging device, it is possible that the observed signal is a result of multiple signals that are behind each other and now are merging into one. To be able to find the main feature points of the signal and to estimate the time of arrival, denoising is required.

There are two types of signals. One appears in the artery and vein region and they have high Signal-to-Noise ratio (SNR) since both of these vessels consume large quantities of blood. The other type is the capillary region where SNR is much lower due to small amount of contrast that enters such region. Examples of such signals are depicted in Fig. 4. The biggest issue in designing the denoising filter is how to effectively model the noise. Normally all imaging devices observe speckle noise which has the Poisson distribution. We found reasonable the assumption that since our images were not fluoroscopic but rather high intensity ones that the number of emitted particles is large enough that we can approximate Poisson distribution with the Gaussian.
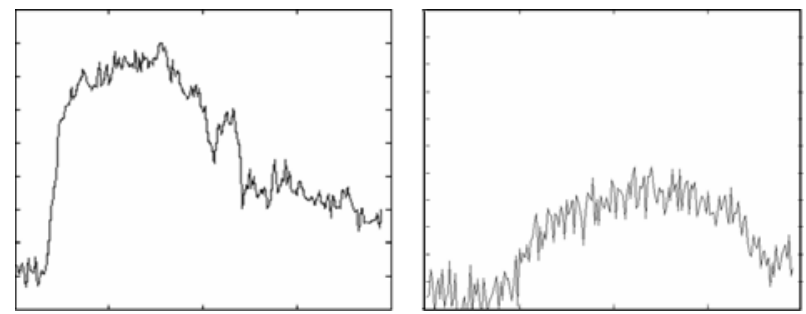

Fig. 4. Typical time density signal in the artery ROI (left) and in the capillary ROI (right) 


\subsection{Wiener Filtering}

Each 1D time-density signal can be viewed as a realization of a stochastic process. We model noise as an additive white Gaussian noise, uncorrelated with the signal i.e. independent and identically distributed (i.i.d.). The stochastic process modeling the signal without noise has low-pass nature due to the fact that timedensity signals are slowly changing functions. The power spectrum of the signal corrupted by noise is a wide band process where the values of higher frequency components are due to noise. So we can estimate noise from the higher frequency part of the signal power spectrum. Since power spectrum of the white noise is flat, the high frequency part is equal to the whole power spectrum. We estimate the noise power spectrum from the highest $50 \%$ of the power spectrum samples of the original signal.

By subtracting the power of noise from the original power spectrum, what remains is the power spectrum of a cleaned version of our original signal (Fig [5]). So the transfer function which defines the Wiener filter is given by:

$$
H_{\text {wiener }}=\frac{S_{s i g}-S_{n n}}{S_{s i g}} .
$$

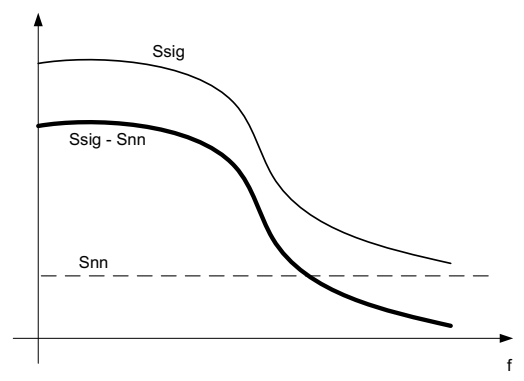

Fig. 5. Power spectrum subtraction

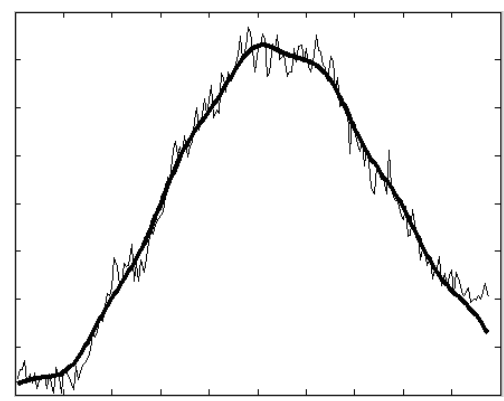

Fig. 6. Wiener denoising of artery ROI 


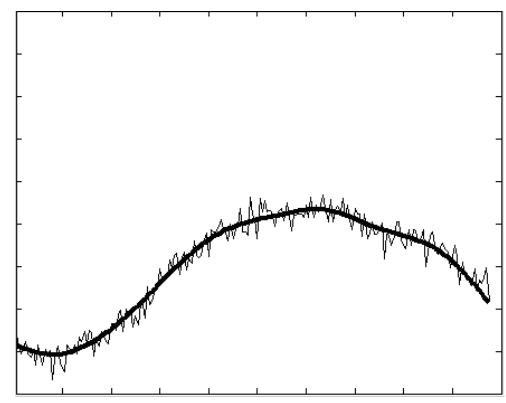

Fig. 7. Wiener denoising of capillary ROI

Results of the filtering in two regions are shown in Fig. 6] and Fig. 7. The resulting denoised time-density curves turned out to be satisfying although improvements are possible.

Additionally, spatial smoothing can be performed by growing the regions of interest. Then, such regions are composed of multiple pixels of the original input image, and are average representatives of these pixels. Spatial smoothing is desirable because it is reasonable to expect that neighboring pixels will have the similar time of arrival. Side-effect of spatial smoothing is the blurred perception of the image since the edges between the vessels and the background are also smoothed out.

\subsection{Wavelet Denoising}

In order to evade the image blurring effect we turned towards the wavelet oriented denoising. Denoising is one of the most important applications of wavelets. Wavelet denoising should not be considered a smoothing method because smoothing always eliminates the high frequency part and keeps the low frequency one. By observing the signal at different scales wavelets are possible to remove the noise without destroying all the high frequency information.

Denoising via wavelet shrinkage method was first suggested by [3. It involves three steps: a linear forward wavelet transform, nonlinear shrinkage of the wavelet coefficients in the wavelet domain and a linear inverse wavelet transform. Wavelet transform has a nice property of de-correlating the signal. Meaning that due to its compact base the signal will be presented by only few non-zero coefficients in the wavelet domain. On the other hand noise which is not correlated will be distributed throughout the wavelet domain in every scale and the corresponding wavelet coefficients will be small because orthogonal wavelet transform preserves the energy of the signal. This means that the wavelet coefficients of a noisy signal are also noisy and if we could eliminate the noise in the wavelet domain, we would also eliminate it in the time domain. Since wavelet coefficients corresponding to noise are small in value and coefficients corresponding to the signal are large, simple thresholding would perform denoising successfully. 


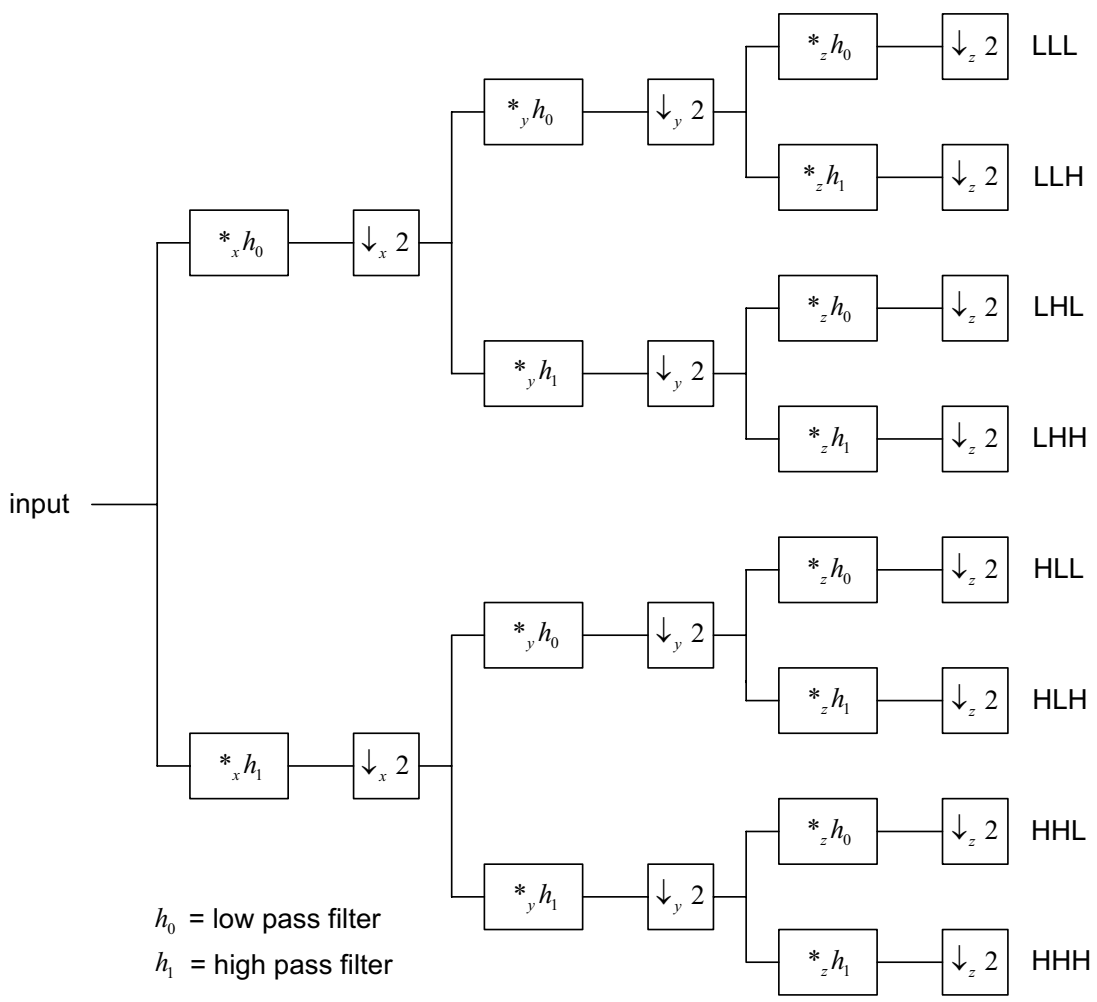

Fig. 8. 3D separable wavelet decomposition

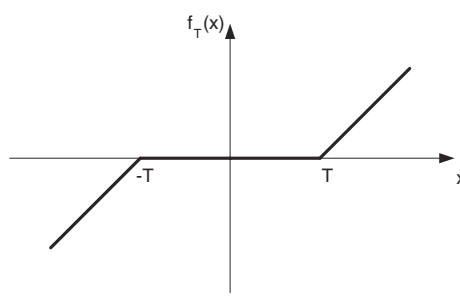

Again we assume additive white Gaussian noise model which is i.i.d. To be able to perform denoising simultaneously in both the time and the spatial domain we opted for 3D orthogonal wavelet transform based on Daubechies db2 wavelet (corresponding filters have 4 coefficients). Such 3D wavelet transform is calculated in a separable man-

Fig. 9. Soft thresholding ner (1D wavelet transform in each dimension). In general, each 3D wavelet decomposition produces 8 sub-volumes as shown in the following Fig. 8 , We used soft thresholding 4 technique and we chose universal threshold [3. Function for performing the soft thresholding with particular threshold $\mathrm{T}$ is shown in Fig. 9 .

The threshold value is not changed between the subbands, hence the name "universal". The universal threshold is estimated from the lowest scale because it is usually the noisiest subband. In our case that is $H H H_{1}$ subband. Although there are numerous other methods for obtaining the threshold parameter which 
are subband adaptive we found that the universal thresholding performed good enough. Universal threshold $\lambda_{\text {univ }}$ is found by the following formula:

$$
\lambda_{\text {univ }}=\sigma \cdot \sqrt{2 \ln (N)} .
$$

$\mathrm{N}$ is the signal length and $\sigma$ is the standard deviation of the noise. $\sigma$ is estimated from the $\mathrm{HHH}_{1}$ subband coefficients by:

$$
\sigma=\operatorname{median}\left(\left|H H H_{1}\right|\right) / 0.6745
$$

The value 0.6745 comes from the median of white gaussian noise with distribution $\mathcal{N}(0,1)$, so the estimator is calibrated for noise with normal distributions. The fact that the $\sigma$ is estimated quite effectively is shown in Fig. 10 and Fig. 11.

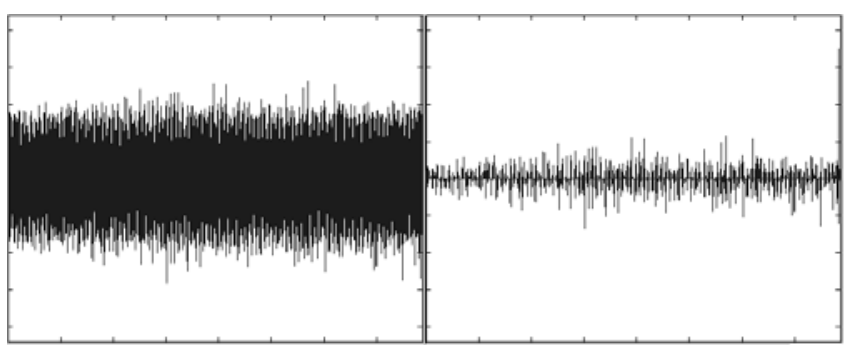

Fig. 10. $H H H_{1}$ wavelet coefficients before (left) and after (right) thresholding

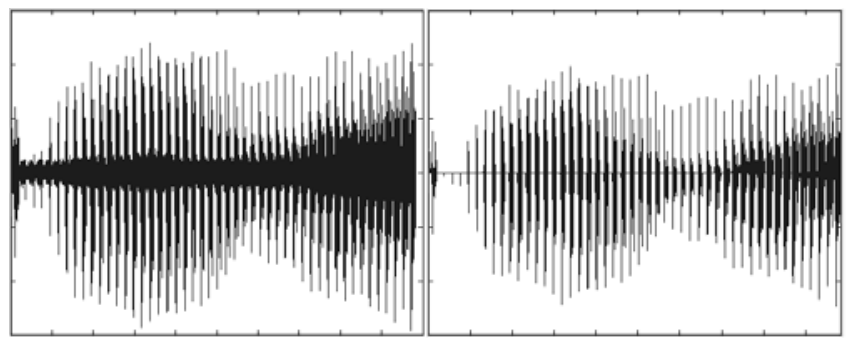

Fig. 11. $H L L_{2}$ wavelet coefficients before (left) and after (right) thresholding

We performed four levels of decomposition and applied soft thresholding to all the detail coefficients. The example of a resulting $1 \mathrm{D}$ time signal is shown in Fig. 12, The denoised signal in Fig. 12 is not as smooth as the wiener denoised one but it is more correlated to the neighboring time-density curve due to simultaneous 2D spatial denoising.

The contribution of $2 \mathrm{D}$ denoising is more evident when displaying the results of the time-of-arrival estimation which will be discussed in the next section. 


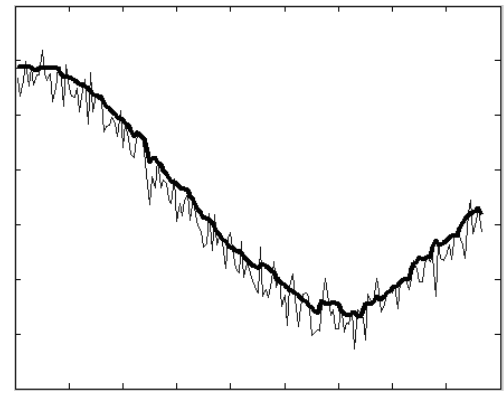

Fig. 12. Signal in the capillary ROI after 3D denoising

\section{$5 \quad$ Results}

The resulting images showing the time of arrival can now be presented for different denoising schemes. The results will be compared to discover the preferred denoising technique. The biggest problem with the most of the medical image analysis methods is the lack of the ground truth. We have the same problem here and currently we can only visually assess the quality of the results. Results for 1D wiener filtering and 3D wavelet denoising are presented in Fig. 13 By observing the resulting images we can notice the following. Wiener filtering method gives results which still contain a lot of noise i.e. resulting neighboring pixels are not correlated enough. The results of wavelet denoising are smoother and the edges are preserved so the images are not blurred.
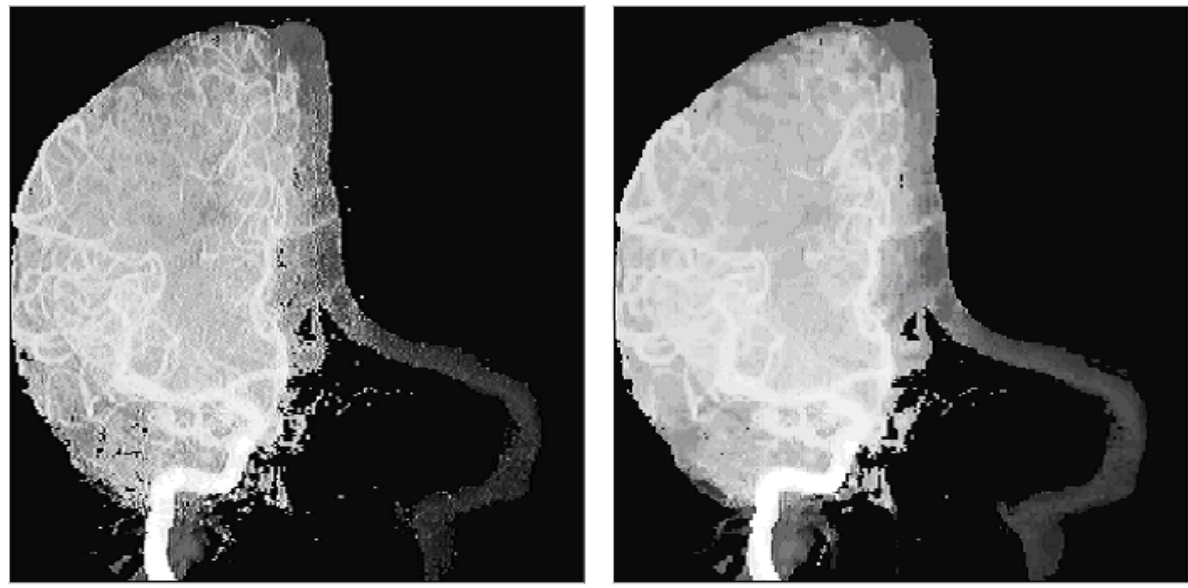

Fig. 13. Result for the Time of Arrival (brighter intensity means shorter time of arrival) after Wiener denoising (left) and Wavelet denoising (right) 


\section{Conclusion}

Different techniques for the denoising of DSA image sequence for calculating the time of arrival of the injected contrast agent are presented. Time dimension contains slowly changing signals so smoothing may be sufficient, but in spatial dimension smoothing leads to the blurred perception. Wiener filtering of $1 \mathrm{D}$ time-density curves enables the estimation of time-of-arrival but the resulting $2 \mathrm{D}$ images are too noisy. On the other hand 3D wavelet non-linear based denoising performs both the time and the spatial denoising and thus gives better results but for the cost of larger computational complexity.

\section{Acknowledgments}

The authors wish to thank Robert Homan for helpful discussions and Dr. Jacques Moret, from the Fondation Rothschild, Paris, for providing us with the image sequences of the brain.

\section{References}

1. Hasegawa, B.: The Physics of Medical X-Ray Imaging, 2nd edition. Medical Physics Publishing Corporation. (1987)

2. Konig, M.: Brain perfusion CT in acute stroke: current status. European Journal of Radiology. 45 (2003) S11-S22.

3. Donoho, D., Johnstone, I.: Ideal adaptation via wavelet shrinkage. Biometrica. 81 (1994) 425-455.

4. Donoho, D.: Denoising by soft thresholding. IEEE Transactions on Information Theory. 41(3) (1995) 613-627. 